\title{
Test of Tube Dilation Picture at Short Times through Dynamic Scattering and Viscoelastic Experiments
}

\author{
Alok K. R. PAUl and Hiroshi WATANABE ${ }^{\dagger}$ \\ Institute for Chemical Research, Kyoto University, Uji, Kyoto 611-0011, Japan
}

(Received July 22, 2003; Accepted October 23, 2003)

\begin{abstract}
The dynamic tube dilation (DTD) concept is tested for the viscoelastic relaxation function $\mu(t)$ and the coherent intermediate scattering function $I_{\mathrm{c}}(\boldsymbol{q}, t)(\boldsymbol{q}=$ the wave vector $)$ of highly entangled linear chains. The analysis made for $I_{\mathrm{c}}$ and $\mu$ involves only the Gaussian nature of the chain and no other details of the chain dynamics. Simple relationships are derived between $I_{\mathrm{c}}(\boldsymbol{q}, t)$ and $\mu(t)$ for the two cases of the fixed and dilated tubes. The range of time considered is longer than the Rouse time $\tau_{\mathrm{R}}$ for contour length fluctuation but smaller than the terminal viscoelastic relaxation time $\tau_{\mathrm{G}}$, and the range of $\mu(t)$ is $1>\mu(t)>0.5$. The relationships are significantly different for the two cases. This difference should enable a test of the DTD picture at short $t$ (but still longer than $\tau_{\mathrm{R}}$ ) through comparison of the $I_{\mathrm{c}}(\boldsymbol{q}, t)$ and $\mu(t)$ data.

KEY WORDS Entanglement / Tube Model / Tube Dilation Mechanism / Dynamic Scattering Function / Viscoelastic Relaxation Function /
\end{abstract}

The dynamic tube dilation (DTD $)^{1}$ mechanism is one of the most fundamental to the current understanding of the entangled chains. ${ }^{2}$ The idea is to replace the difficult many-body problem of the dynamics in concentrated polymer systems with a tractable single body problem in an effective field. The 'single body' in this case is the single polymer chain and the effective field is a dilating tube-like region of topological constraints surrounding the chain contour. This is a phenomenological description of the non-crossability of chains and the mutual equilibration of successive entanglement segments in a given chain. The DTD process is activated by the constraint-release- (CR-) induced motion i.e., by movement of the surrounding chains (see refs. 2 and 3 for example).

The chain motion beyond the entanglement lengthscale has been successfully treated by the topological notion of the tube and by incorporating various modes of motion like the contour length fluctuation (CLF). The theory considering DTD could satisfactorily predict the linear viscoelastic behavior of entangled monodisperse chains. ${ }^{3-6}$ However, the viscoelastic function represents an orientational anisotropy at a given time $^{7}$ and fails to probe the correlation in the dynamics of the chain at two seperate times. In other words, the agreement of the DTD theory with the viscoelastic data does not testify that the chain actually moves in the way assumed in the model. ${ }^{2}$ It is important to study other dynamic properties that differently average the stochastic motion of the chain.

In the DTD picture, the relaxed portion of the chain has been regarded as a solvent. ${ }^{1}$ This solvent dilates the tube diameter with time as $a^{\prime}(t)=a\left[\varphi^{\prime}(t)\right]^{-\alpha / 2}$.
Here $a$ is the diameter of the non-dilated tube, $\varphi^{\prime}(t)$ is the survival fraction of the dilated tube at $t$, and $\alpha$ $(=1-1.3)$ is a dilation exponent. In order to test the DTD picture we derived a relationship between the normalized dielectric relaxation function $\Phi(t)$ and viscoelastic relaxation function $\mu(t)$ for type-A chains having parallel dipoles ${ }^{8-11}$

$$
\mu(t)=[\Phi(t)]^{1+\alpha}(+ \text { tube-edge correction term }) .
$$

This was derived assuming only the Gaussian nature of the chain and irrespective of the detailed dynamics. Studies of the viscoelastic and dielectric data ${ }^{8-12}$ revealed the general validity of eq. 1 for representative type-A chains, monodisperse linear cis-polyisoprene (PI) chains, at long $t$. Thus the monodisperse linear chain quite possibly moves in the dilated tube of diameter $a^{\prime}$ in the terminal relaxation regime.

However, the situation at short times is rather intriguing. Though the chain still has, on an average, the equilibrium contour length, one cannot help suspecting if the DTD picture holds valid in the time scales of the order of the Rouse time $\tau_{R}$. At short times, the chain segments exhibit a short-ranged motion, which may lead to an insufficient dilation. Hence, even for monodisperse linear chains, the DTD picture may fail at short $t$.

In principle, this failure at short $t$ can be examined by testing the validity of eq. 1 for the viscoelastic $\mu(t)$ and dielectric $\Phi(t)$ data. However, we face a practical difficulty. While eq. 1 holds for the relaxation due to the global chain motion, the measured $\mu(t)$ and $\Phi(t)$ data at short $t$ contains, in addition, a contribution

${ }^{\dagger}$ To whom correspondence should be addressed (E-mail: hiroshi@scl.kyoto-u.ac.jp). 
due to the fast local motion of the monomeric segments within the entanglement segment. The subtraction of these contributions is crucial for the above test.

For the $\mu(t)$ data, the modified stress-optical rule (MSOR) ${ }^{13,14}$ may be used to subtract the contribution of the local motion, and the relaxation only due to the global chain motion can be evaluated with satisfactory accuracy. However, a similarly accurate subtraction for dielectric data is rather cumbersome. For most of type-A chains at short $t$ the local motion of the monomeric segments is dielectrically active because of their type-B dipoles perpendicular to the chain backbone. Consequently, the $\Phi(t)$ obtained after this subtraction always includes a non-negligible uncertainty at short $t$. Further, the normalized relaxation functions $\mu(t)$ and $\Phi(t)$ are close to unity at short $t$, and this uncertainty is disturbing while experimentally distinguishing the two relationships, $\mu(t)=[\Phi(t)]^{1+\alpha}$ (in presence of DTD) and $\mu(t)=\Phi(t)$ (in absence of DTD).

Hence, for a practical test of the DTD picture at short times we should devise experiments relating the viscoelastic and non-dielectric properties, the latter being accurately measurable at short $t$, and formulate their DTD and non-DTD relationships that are experimentally distinguishable. We explored various possibilities and found that the mean-square displacement of the primitive chain segments, $[d(n, t)]^{2}$ (with $n$ $=$ segment index) without the contribution of non-diffusive part of the CLF, can be easily analyzed. Focusing on this feature of $[d(n, t)]^{2}$ we formulated the DTD and non-DTD relationships between $\mu(t)$ and $[d(n, t)]^{2}$ at short $t .{ }^{15}$ The incoherent intermediate scattering function of actual chains, $I_{\mathrm{s}}(\boldsymbol{q}, t)$ with $\boldsymbol{q}$ being the wave vector, can be expressed in terms of this $[d(n, t)]^{2}$, enabling us to test the DTD picture at short $t$ through comparison of the viscoelastic and dynamic scattering data ( $\mu$ and $I_{s}$ ).

As an extension of the previous work, we have focused on the mean-square displacement function $\left[d_{2}\left(n, n^{\prime}, t\right)\right]^{2}=\left\langle\left[\boldsymbol{r}(n, t)-\boldsymbol{r}\left(n^{\prime}, 0\right)\right]^{2}\right\rangle$ defined for different ( $n$-th and $n^{\prime}$-th) segments. We have derived a simple expression of $d_{2}^{2}$ in terms of $n, n^{\prime}$, and $\mu$ at short $t$ by just considering the Gaussian nature of the chain. The coherent intermediate scattering function of actual chains $I_{\mathrm{c}}(\boldsymbol{q}, t)$ is expressed in terms of $d_{2}^{2}$, and the DTD picture can be tested through comparison of the $I_{\mathrm{c}}$ and $\mu$ data. This can serve an even wider route for our test of the DTD picture at short $t$. This paper presents the results.

In the remaining part of the paper, we first define the physical quantities relevant to our analysis. Then, we focus on the evaluation of the displacement function $d_{2}^{2}$ of the primitive segments and formulate relationships between $d_{2}^{2}$ and $\mu(t)$ for the two cases of the fixed and dilated tube. The results are utilized to formulate relationships between coherent intermediate scattering function $I_{\mathrm{c}}(\boldsymbol{q}, t)$ and $\mu(t)$ of the chains for these two cases, and a possible experimental method for testing the DTD picture at short $t$ is presented. Finally we summarize our results with a brief concluding remarks.

\section{MEAN SQUARE DISPLACEMENT AND COHERENT INTERMEDIATE SCATTERING FUNCTION}

We consider a system of highly entangled monodisperse linear Gaussian chains at equilibrium. The topological non-crossing constraints are represented as a tube and the chain is subdivided into primitive segments (entanglement segments) of size $a$. This $a$ is identical to the tube diameter. Then, the real chain is coarse-grained into a primitive chain ${ }^{7}$ that represents a series of connected axes of the primitive segments. Since the system is at equilibrium the meansquare end-to-end distance of the primitive chain is given by $\left\langle R^{2}\right\rangle=N a^{2}$, where $N(\gg 1)$ is the number of the primitive segments per chain.

The time scale $t$ considered here is longer than the Rouse time $\tau_{\mathrm{R}}$ for the thermal contour length fluctuation (CLF) along the non-dilated tube, but shorter than the terminal viscoelastic relaxation time $\tau_{\mathrm{G}}$ to an extent that the Fickian diffusion (giving the segment displacement proportional to $\sqrt{t}$ in 3-dimensional space) negligibly contributes the mean-square displacement function. In this time scale, the real chain can be safely coarse-grained into the primitive chain having a constant contour length $\mathrm{Na}$. Then, the mean-square displacement of the $n$-th primitive segment at time $t$ with respect to the $n^{\prime}$-th segment at time 0 can be written as $\left[\mathscr{D}_{2}\left(n, n^{\prime}, t\right)\right]^{2}=\left[d_{2}\left(n, n^{\prime}, t\right)\right]^{2}+d_{2, \mathrm{CLF}}^{2}$. Here, $d_{2, \mathrm{CLF}}^{2}$ is the contribution to $\mathscr{D}_{2}^{2}$ due to the non-diffusive part of CLF, i.e., the part without the curvilinear diffusion along the tube. The diffusive part is included in $\left[d_{2}\left(n, n^{\prime}, t\right)\right]^{2}$. This $\left[d_{2}\left(n, n^{\prime}, t\right)\right]^{2}$ is defined in terms of the position vector $\boldsymbol{r}(n, t)$ of the $n$-th primitive segment at time $t\left(>\tau_{\mathrm{R}}\right)$ and the position vector $\boldsymbol{r}\left(n^{\prime}, 0\right)$ of the $n^{\prime}$-th primitive segment at time 0 ,

$$
\left[d_{2}\left(n, n^{\prime}, t\right)\right]^{2}=\left\langle\left[\boldsymbol{r}(n, t)-\boldsymbol{r}\left(n^{\prime}, 0\right)\right]^{2}\right\rangle .
$$

Here, $\langle\ldots\rangle$ represents an ensemble average taken for the primitive chains having the constant contour length $\mathrm{Na}$.

In Figure 1, we illustrate the movement of the primitive chain. The dashed and the solid curves represent the primitive chain at times 0 and $t$, respectively. The parts $a$ and $b$ schematically show the changes of the conformation of the chain trapped in a spatially fixed (non-dilated) tube, and the parts $\mathrm{c}$ and $\mathrm{d}$ indicate the 
(a)

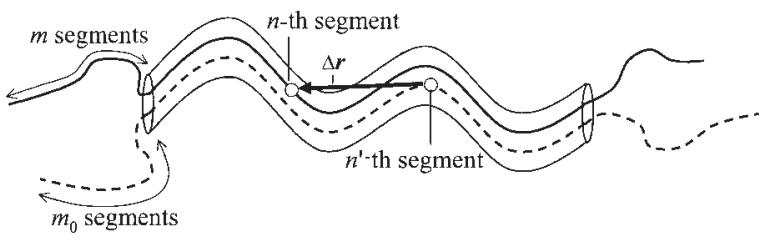

(b)

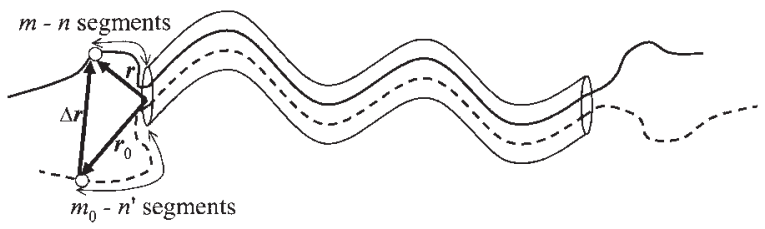

(c)

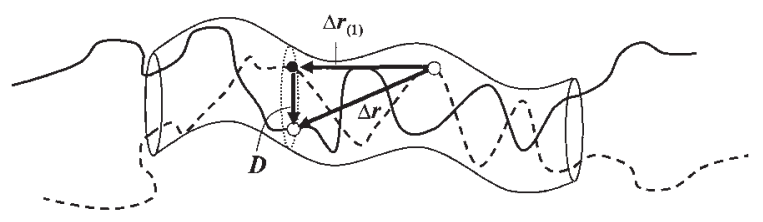

(d)

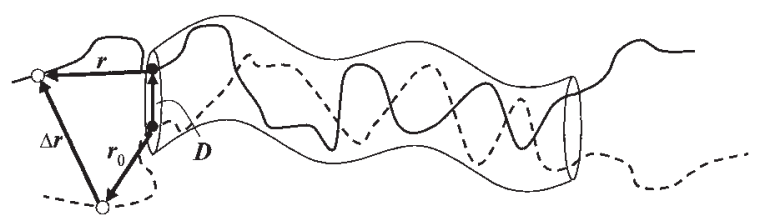

Figure 1. Schematic illustration of the primitive chains moving in the fixed tube (parts a and b) and in the dilated tube (parts $c$ and d). The dashed and solid curves represent the chain at times 0 and $t$, respectively. The arrow represents the displacement $\Delta \boldsymbol{r}$ for the $n$-th segment at time $t$ with respect to the $n^{\prime}$-th segment at time 0 . The contribution of the contour length fluctuation is not included in this $\Delta r$.

conformational changes for the case of DTD. The displacement vector $\Delta \boldsymbol{r}=\boldsymbol{r}(n, t)-\boldsymbol{r}\left(n^{\prime}, 0\right)$ for both fixed tube and DTD cases is shown.

As can be noted from these, $\left[d_{2}\left(n, n^{\prime}, t\right)\right]^{2}$ reflects the longitudinal motion of the chain along the fixed/ dilated tube and also the lateral motion in the dilated tube. Considering the Gaussian feature of the chain, we can characterize $\Delta \boldsymbol{r}$ without CLF in terms of the survival fraction of either the fixed or dilated tube. The survival fraction in both cases can be related to the viscoelastic relaxation function $\mu(t)$. Hence we obtain a relationship between $\mu(t)$ and $\left[d_{2}\left(n, n^{\prime}, t\right)\right]^{2}$, as explained later in details.

The coherent intermediate scattering function $I_{\mathrm{c}}(\boldsymbol{q}, t)$ reflects motion of the scattering units (i.e. monomeric segments) in the real chain. We focus on a real (non-primitive) chain having the scattering label such as the isotope label. This labeled chain is a dilute component placed in a matrix of unlabeled chains of the same molecular weight. Thus, $I_{\mathrm{c}}(\boldsymbol{q}, t)$ at the wave vector $\boldsymbol{q}$ at time $t$ detects the motional correlation within this labeled chain (probe) in the monodisperse system and can be expressed as ${ }^{16}$

$$
\begin{array}{r}
I_{\mathrm{c}}(\boldsymbol{q}, t)=\frac{\sum_{j=1}^{g N} \sum_{j^{\prime}=1}^{g N} \vartheta_{j} \vartheta_{j}^{\prime}\left\langle\exp \left\{i \boldsymbol{q} \cdot\left[\rho(j, t)-\rho\left(j^{\prime}, 0\right)\right]\right\}\right\rangle}{\sum_{j=1}^{g N} \vartheta_{j}} \\
(i=\sqrt{-1})
\end{array}
$$

Here, $\rho(j, t)$ and $\rho\left(j^{\prime}, 0\right)$ are the positions of the $j$-th and $j^{\prime}$-th monomeric segments at the times $t$ and 0 , respectively, and $\vartheta_{j}$ is a parameter specifying the labeling $\left(\vartheta_{j}=1\right.$ and 0 for the labeled and unlabeled monomeric segments in the probe chain). The indices $j$ and $j^{\prime}$ run from 1 to $g N$, where $g$ is the number of the monomeric segments per primitive segment.

In this paper we make no detailed assumption about the chain motion determining $d_{2}^{2}$ to ensure the maximum generality of the obtained relationships between $\mu$ and $I_{\mathrm{c}}$. (If we assume a time evolution equation for the primitive segment position, we can explicitly calculate $I_{\mathrm{c}}$ and $\mu$ as functions of $t$ and obtain their relationship by erasing $t$. However, we do not adopt this approach so that the uncertainty due to this assumption is avoided.)

In principle, the tube survival fraction has a distribution among the chains in the system. However, in the analysis presented in this paper, the survival fraction is regarded to be the same for all probe chains. This is the largest approximation in this paper.

Our aim is to formulate the relationship between $\mu(t)$ and $I_{\mathrm{c}}(\boldsymbol{q}, t)$ for both cases of presence and absence of DTD and also to discuss an experimental mode for the test of the DTD picture at short $t$. The formulation is based on the derivation of the mean-square displacement function $\left[d_{2}\left(n, n^{\prime}, t\right)\right]^{2}$ for both cases. In the remaining part of the paper, we derive the relationship between $\mu(t)$ and $\left[d_{2}\left(n, n^{\prime}, t\right)\right]^{2}$ for the case of the fixed tube and then for the case of DTD. The results are used to obtain the relationships between $I_{\mathrm{c}}(\boldsymbol{q}, t)$ and $\mu(t)$ for both cases.

\section{RELATIONSHIP BETWEEN $\left[d_{2}\left(n, n^{\prime}, t\right)\right]^{2}$ AND $\mu(t)$ FOR THE PROBE CHAIN IN A FIXED TUBE}

Consider two snapshots of the primitive chain in a fixed tube at times 0 and $t$. The chain at these times has an overlapping in the portion of the tube surviving at $t$; see the dashed and solid curves in Figures 1a and $1 b$. At these times, the same number $(N \varphi)$ of the primitive segments are contained in this portion having the survival fraction $\varphi$. Under this situation, the chain conformations at the times 0 and $t$ can be characterized by the numbers $m_{0}$ and $m$ of the primitive segments out of the left edge of the surviving portion at these times; see Figure 1a. The $m_{0}$ and $m$ values have a distribution in a range between 0 and $N(1-\varphi)$.

In Figures $1 \mathrm{a}$ and $1 \mathrm{~b}$, the $n$-th and $n^{\prime}$-th primitive segments are shown with the unfilled circles (with 
the indices $n$ and $n^{\prime}$ defined with respect to the left end of the chain). The displacement vector $\Delta \boldsymbol{r}=[\boldsymbol{r}(n, t)-$ $\left.\boldsymbol{r}\left(n^{\prime}, 0\right)\right]$ is geometrically determined by the $m, m_{0}, n$ and $n^{\prime}$ values. For $t$ well below the terminal viscoelastic relaxation time $\tau_{\mathrm{G}}$, the Fickian diffusion of the chain is negligible and the average of $(\Delta r)^{2}$ is classified into some cases explained below.

Case 1: For $0 \leq n^{\prime} \leq m_{0}$ and $0 \leq n \leq m$, the $n$-th and $n^{\prime}$-th primitive segments at the times $t$ and 0 are out of the left edge of the surviving portion of the tube, see Figure $1 \mathrm{~b}$. Then, $\Delta \boldsymbol{r}$ is given by $\boldsymbol{r}-\boldsymbol{r}_{0}$, where $\boldsymbol{r}$ and $\boldsymbol{r}_{0}$ are the vectors connecting the center of the tube edge and the segments $n$ and $n^{\prime}$ at these times. Because of the Gaussian feature of the chains, the vectors $\boldsymbol{r}$ and $\boldsymbol{r}_{0}$ for the sequences of $m-n$ and $m_{0}-n^{\prime}$ segments are uncorrelated with each other. Thus, $\Delta \boldsymbol{r}$ is equivalent to an end-to-end vector of a hypothetical Gaussian sub-chain composed of $(m-n)+\left(m_{0}-n^{\prime}\right)$ segments, and the conditional average of $(\Delta \boldsymbol{r})^{2}$ evaluated for all conformations of this sub-chain under a constraint of the fixed $m, m_{0}$, $n$ and $n^{\prime}$ values is written as

$$
\left[\overline{(\Delta \boldsymbol{r})^{2}}\right]_{\text {case } 1}=a^{2}\left(m+m_{0}-n-n^{\prime}\right) .
$$

Case 2: For $m_{0}+N \varphi<n^{\prime} \leq N$ and $m+N \varphi<$ $n \leq N$, the $n$-th and $n^{\prime}$-th primitive segments at the times $t$ and 0 are out of the right edge of the surviving portion of the tube. This situation is equivalent to that in the case (1), and the conditional average of $(\Delta r)^{2}$ is found to be

$$
\left[\overline{(\Delta \boldsymbol{r})^{2}}\right]_{\text {case } 2}=a^{2}\left(n+n^{\prime}-m-m_{0}-2 N \varphi\right) .
$$

Case 3: For $m_{0} \leq n^{\prime} \leq m_{0}+N \varphi$ and $m \leq n \leq m+$ $N \varphi$, the $n$-th and $n^{\prime}$-th primitive segments at the times $t$ and 0 are in the surviving portion of the tube; see Figure 1a. Then, $\Delta \boldsymbol{r}$ is equivalent to an end-to-end vector of a portion of the chain composed of $\left|(n-m)-\left(n^{\prime}-m_{0}\right)\right|$ segments, and the conditional average of $(\Delta \boldsymbol{r})^{2}$ is given by

$$
\left[\overline{(\Delta \boldsymbol{r})^{2}}\right]_{\text {case } 3}=a^{2}\left|(n-m)-\left(n^{\prime}-m_{0}\right)\right| .
$$

Case 4: For the remaining sets of $m, m_{0}, n$ and $n^{\prime}$ values, the $n$-th primitive segment at time $t$ is in the surviving portion of the tube while the $n^{\prime}$-th segment at time 0 is outside this portion, or vice versa. A simple geometrical consideration gives the conditional average of $(\Delta \boldsymbol{r})^{2}$ in this case. For example, for $n<m$ and $m_{0}<n^{\prime}<m_{0}+N \varphi, \Delta \boldsymbol{r}$ is given by a difference of the end-to-end vectors of two sequences composed of $m-n$ segments and $n^{\prime}-m_{0}$ segments, with both vectors starting from the center of the left edge of the surviving portion of the tube. This $\Delta \boldsymbol{r}$ is equivalent to an end-to-end vector of a hypothetical Gaussian sub-chain composed of $\mid(m-n)-$ $\left(m_{0}-n^{\prime}\right) \mid$ segments, and the conditional average $(\Delta \boldsymbol{r})^{2}$ is given by $a^{2}\left|(m-n)-\left(m_{0}-n^{\prime}\right)\right|$. From similar considerations for all values of $m, m_{0}, n$ and $n^{\prime}$, we find

$$
\left[\overline{(\Delta \boldsymbol{r})^{2}}\right]_{\text {case } 4}=a^{2}\left|(m-n)-\left(m_{0}-n^{\prime}\right)\right| .
$$

\section{Expression of $\left[d_{2}\left(n, n^{\prime}, t\right)\right]^{2}:$ Asymptotic Analysis}

For a given survival fraction of the fixed tube, $\varphi$, the $m$ and $m_{0}$ are equivalent to each other and have the same distribution described by a distribution function $\Psi(\xi)$ defined for $\xi=m$ or $m_{0}$ in a range of $0 \leq$ $\xi \leq N(1-\varphi) .{ }^{15}$ This $\Psi$ is normalized to satisfy $\int_{0}^{\overline{N(1-\varphi)}} \mathrm{d} \xi \Psi(\xi)=1$.

Utilizing this $\Psi$, we can express the mean-square displacement function defined in eq. 2 as

$$
\begin{aligned}
{\left[d_{2}\left(n, n^{\prime}, t\right)\right]^{2}=} & \int_{0}^{N(1-\varphi)} \int_{0}^{N(1-\varphi)} \\
& \times \mathrm{d} m \mathrm{~d} m_{0} \Psi(m) \Psi\left(m_{0}\right)\left[\overline{(\Delta \boldsymbol{r})^{2}}\right] .
\end{aligned}
$$

where $\left[\overline{(\Delta \boldsymbol{r})^{2}}\right]$ represents conditional averages for the cases 1-4 explained above. Among the four cases, more than two cases may emerge for different $m$ and/or $m_{0}$ values in the integration range in eq. 8, and the $\left[\overline{(\Delta \boldsymbol{r})^{2}}\right]$ is chosen from those given by eqs. 4-7 according to the $n, n^{\prime}, m$ and $m_{0}$ values.

The $\left[d_{2}\left(n, n^{\prime}, t\right)\right]^{2}$ can be unequivocally calculated if the functional form of $\Psi(m)$ is known; cf. eq. 8. However, before considering this form, we examine general features of $\left[d_{2}\left(n, n^{\prime}, t\right)\right]^{2}$ that can be deduced from eq. 8 without a detailed knowledge about $\Psi(m)$.

For example, for the self-displacement of the endsegment (for $n=n^{\prime}=0$ or $n=n^{\prime}=N$ ), only case 1 or case 2 is allowed. Then, eq. 8 is simplified to $\left[d_{2}(0,0, t)\right]^{2}=a^{2}\left(\langle m\rangle+\left\langle m_{0}\right\rangle\right)$ and $\left[d_{2}(N, N, t)\right]^{2}=$ $a^{2}\left[2 N(1-\varphi)-\langle m\rangle-\left\langle m_{0}\right\rangle\right] \quad$ with $\quad\langle m\rangle=\left\langle m_{0}\right\rangle=$ $\int_{0}^{N(1-\varphi)} \mathrm{d} m m \Psi(m)$. Since the two ends of the chains are equivalent, the chain should have, on average, the same number of the segments out of the left and right edges of the surviving portion of the tube. Thus, irrespective of the details of $\Psi(m)$, the average number is given by $\langle m\rangle=\left\langle m_{0}\right\rangle=N(1-\varphi) / 2$. (Note that $N(1-\varphi)$ is the total number of the segments out of the surviving portion.) From this result, we obtain ${ }^{15}$

$$
d_{2}^{2}=\left\langle R^{2}\right\rangle(1-\varphi(t))
$$

(for the self-displacement of the end-segment)

Here, $\left\langle R^{2}\right\rangle\left(=N a^{2}\right)$ is the mean-square end-to-end distance of the chain.

The other example is found for the self-displacement of the segment at the middle of the chain $\left(n=n^{\prime}=N / 2\right)$. For this segment, all four cases $1-4$ are allowed in the integration range in eq. 8 if $\varphi$ is smaller than 0.5. Thus, for $\varphi<0.5$, an exact calcula- 
tion of $d_{2}^{2}$ requires detailed information about $\Psi(m)$. In contrast, for $\varphi>0.5$, only the case 3 is allowed and eq. 8 is reduced to

$$
\left[d_{2}(N / 2, N / 2, t)\right]^{2}=a^{2}\left\langle\left|m-m_{0}\right|\right\rangle
$$

with

$$
\begin{aligned}
\left\langle\left|m-m_{0}\right|\right\rangle= & \int_{0}^{N(1-\varphi)} \int_{0}^{N(1-\varphi)} \\
& \times \mathrm{d} m \mathrm{~d} m_{0} \Psi(m) \Psi\left(m_{0}\right)\left|m-m_{0}\right|
\end{aligned}
$$

Since the variable $\left|m-m_{0}\right|$ appearing in eqs. 10 and 11 is equivalent to an absolute value of the curvilinear displacement along the fixed tube, the average $\left\langle\left|m-m_{0}\right|\right\rangle$ corresponds to the abandoned number of the initial tube segments at the left (or the right) side of the surviving portion of the tube. Thus, the average can be safely approximated as $\left\langle\left|m-m_{0}\right|\right\rangle \cong$ $N(1-\varphi) / 2$, and eq. 10 becomes

$$
d_{2}^{2}=\left\langle R^{2}\right\rangle(1-\varphi(t)) / 2 \quad \text { (for the middle-segment) }
$$

In fact, eq. 12 excellently describes $d_{2}^{2}$ of the middle segment for the chain reptating in the fixed tube. ${ }^{15}$

For the cross-displacement of particular segments, we can also find simple expressions of $d_{2}^{2}$. For example, for $n=N / 2$ and $n^{\prime}=0$, the $n$-th and $n^{\prime}$-th primitive segments, respectively, are inside and outside of the surviving portion of the tube irrespective of the $m_{0}$ and $m$ values. Hence the only applicable conditional average is that of case 4 . Then, eq. 8 is simplified as

$$
\left[d_{2}(N / 2,0, t)\right]^{2}=a^{2}\left(N / 2-\langle m\rangle+\left\langle m_{0}\right\rangle\right)=a^{2} N / 2
$$

Similarly, we have $\left[d_{2}(0, N / 2, t)\right]^{2}=a^{2} N / 2$.

We also find that for $n^{\prime}=0$ and $n=N$ only applicable conditional average is that of case 4 and eq. 8 is simplified to

$$
\left[d_{2}(N, 0, t)\right]^{2}=a^{2}\left(N-\langle m\rangle+\left\langle m_{0}\right\rangle\right)=a^{2} N
$$

Similarly, $\left[d_{2}(0, N, t)\right]^{2}=a^{2} N$.

Approximate Expression of $\left[d_{2}\left(n, n^{\prime}, t\right)\right]^{2}$ in the Entire Range of $n$ and $n^{\prime}$

For $\varphi(t)>0.5$, the $\left[d_{2}\left(n, n^{\prime}, t\right)\right]^{2}$ calculated from eq. 8 is classified into three regimes specified below according to the $n$ and $n^{\prime}$ values:

Regime i: $0 \leq n, n^{\prime}<N(1-\varphi)$

$$
\left[d_{2}\left(n, n^{\prime}, t\right)\right]^{2} / a^{2}=\left\langle\left|(n-m)-\left(n^{\prime}-m_{0}\right)\right|\right\rangle+I
$$

with

$$
I=\int_{n}^{N(1-\varphi)} \int_{n^{\prime}}^{N(1-\varphi)} \mathrm{d} m \mathrm{~d} m_{0} \Psi(m) \Psi\left(m_{0}\right)\left[m_{0}-n^{\prime}+m-n-\left|(n-m)-\left(n^{\prime}-m_{0}\right)\right|\right]
$$

Regime ii: $N \varphi<n, n^{\prime} \leq N$

$$
\left[d_{2}\left(n, n^{\prime}, t\right)\right]^{2} / a^{2}=\left\langle\left|(n-m)-\left(n^{\prime}-m_{0}\right)\right|\right\rangle+I I
$$

with

$$
I I=\int_{0}^{n-N \varphi} \int_{0}^{n^{\prime}-N \varphi} \mathrm{d} m \mathrm{~d} m_{0} \Psi(m) \Psi\left(m_{0}\right)\left[n^{\prime}-m_{0}+n-m-2 N \varphi-\left|(n-m)-\left(n^{\prime}-m_{0}\right)\right|\right]
$$

Regime iii: For all other $n, n^{\prime}$

$$
\left[d_{2}\left(n, n^{\prime}, t\right)\right]^{2} / a^{2}=\left\langle\left|(n-m)-\left(n^{\prime}-m_{0}\right)\right|\right\rangle
$$

For all these regimes, the average $\left\langle\left|(n-m)-\left(n^{\prime}-m_{0}\right)\right|\right\rangle$ is defined as

$$
\left\langle\left|(n-m)-\left(n^{\prime}-m_{0}\right)\right|\right\rangle=\int_{0}^{N(1-\varphi)} \int_{0}^{N(1-\varphi)} \mathrm{d} m \mathrm{~d} m_{0} \Psi(m) \Psi\left(m_{0}\right)\left|(n-m)-\left(n^{\prime}-m_{0}\right)\right|
$$

This average as well as the integrals $I$ and $I I$ can be accurately evaluated only when $\Psi(m)$ is explicitly known. However, we note a general, qualitative feature of $\Psi$ : For any thermal (diffusive) motion of the chain along the fixed tube, the number $m$ of the segments out of the left edge of the surviving portion of the tube would be broadly distributed (as is the case for the reptative motion). Then, $\Psi(m)$ would be rather weakly dependent on $m$. Considering this feature of $\Psi(m)$ and the width of the integration ranges in eqs. 16, 18 and 20, we attempted to approximately calculate $d_{2}^{2}$. This approximation was made (partly 
in a trial-and-error fashion) so that the resulting expression of $d_{2}^{2}$ reproduced the expression for the special cases (eqs. 9, 12, 13 and 14) as well as the previously obtained expression of the self-displacement function ${ }^{15} d^{2}(n, t)=d_{2}^{2}(n, n, t)$. Then we casted eqs. 15-19 into an approximate but simple relationship between the mean square cross-displacement function $d_{2}^{2}$ and the viscoelastic relaxation function $\mu(t)$, the latter coinciding with the tube survival fraction $\varphi(t)$ for the case of fixed tube. ${ }^{2,7}$ The results are summarized as

Regime i: $0 \leq \theta, \theta^{\prime}<(1-\mu)$

$$
\begin{aligned}
d_{2}^{2} \cong & \left\langle R^{2}\right\rangle\left[\gamma+\frac{(1-\mu-\theta)\left(1-\mu-\theta^{\prime}\right)}{(1-\mu)^{2}}\right. \\
& \left.\times\left(1-\mu-\frac{\theta+\theta^{\prime}}{2}-\gamma\right)\right]
\end{aligned}
$$

Regime ii: $\mu<\theta, \theta^{\prime} \leq 1$

$d_{2}^{2} \cong\left\langle R^{2}\right\rangle\left[\gamma+\frac{(\theta-\mu)\left(\theta^{\prime}-\mu\right)}{(1-\mu)^{2}}\left(-\mu+\frac{\theta+\theta^{\prime}}{2}-\gamma\right)\right]$

Regime iii: For all other $\theta, \theta^{\prime}$

$$
d_{2}^{2} \cong\left\langle R^{2}\right\rangle \gamma
$$

Here, $\quad \gamma=\left[\left|\theta-\theta^{\prime}\right|^{3}+\{(1-\mu) / 2\}^{3}\right]^{1 / 3}, \quad \theta=n / N$, and $\theta^{\prime}=n^{\prime} / N$. On substituting $\theta=\theta^{\prime}$ we recover the expression of the self-displacement function derived in the earlier paper. ${ }^{15}$

The approximation underlying eqs. 21-23 may look crude. Nonetheless, Figure 2 demonstrates that the $d_{2}^{2}$ given by eqs. 21-23 (curves) is surprisingly close to the $d_{2}^{2}$ of the chain reptating in the fixed tube (symbols). (Some details of the reptation theory are given in Appendix A.) Thus, we can utilize eqs. 21-23 to formulate the $d_{2}^{2}$ for the case of DTD, as described below.

\section{RELATIONSHIP BETWEEN $\left[d_{2}\left(n, n^{\prime}, t\right)\right]^{2}$ AND $\mu(t)$ FOR THE CHAIN IN A DILATED TUBE}

Here, we consider the primitive chain in a dilated tube. This tube have a survival fraction $\varphi^{\prime}(t)$ and a fully dilated diameter ${ }^{1,2} a^{\prime}=a\left[\varphi^{\prime}(t)\right]^{-\alpha / 2}$, with the dilation exponent $\alpha=1-1.3$. In Figures $1 \mathrm{c}$ and $1 \mathrm{~d}$, two snapshots of the primitive chain at times 0 and $t$ are shown with dashed and solid curves, respectively. The number of the primitive segments in the surviving portion of the dilated tube is $N \varphi^{\prime}(t)$. We limit ourselves in the range of $\varphi^{\prime}(t)>0.5$ so that the results obtained for the fixed tube case can be utilized for the calculation of $\left[d_{2}\left(n, n^{\prime}, t\right)\right]^{2}$ for the DTD case. For highly entangled linear chains, this range of $\varphi^{\prime}$ (as
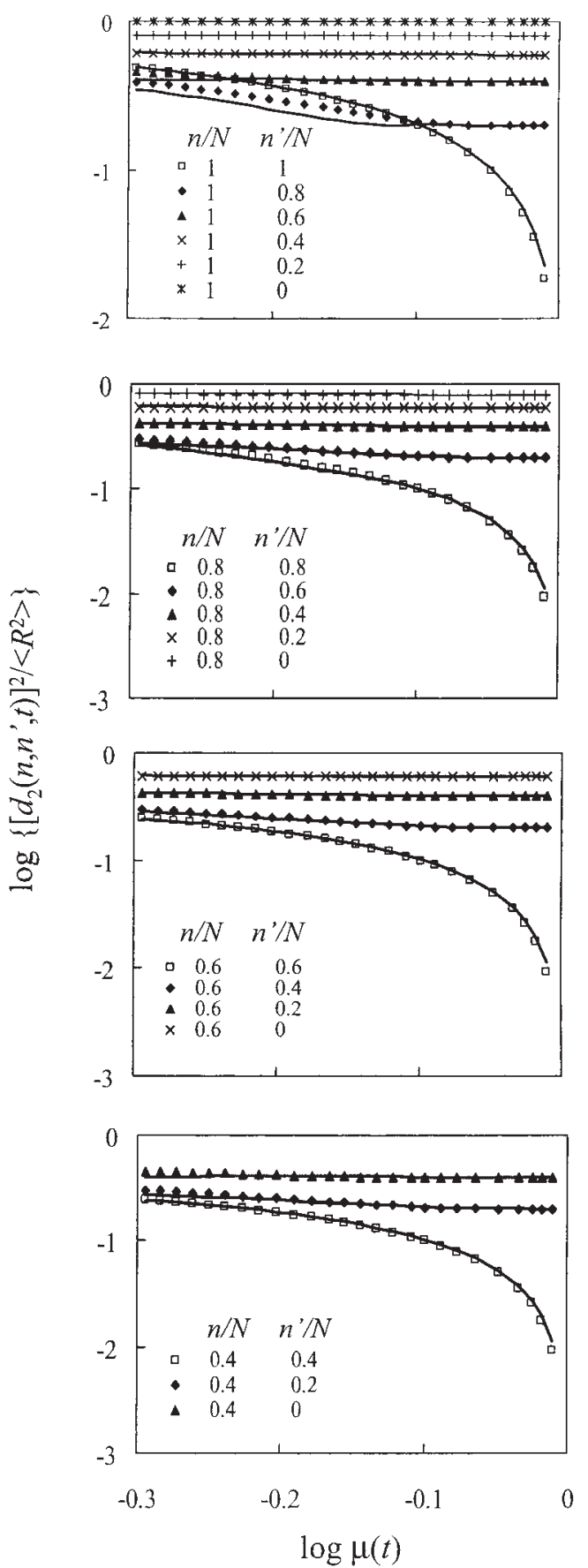

Figure 2. Plots of the normalized cross-displacement of the $n$ th primitive segment with respect to $n^{\prime}$-th primitive segment at time $0,\left[d_{2}\left(n, n^{\prime}, t\right)\right]^{2} /\left\langle R^{2}\right\rangle$ against the normalized viscoelastic relaxation function $\mu(t)$ for the chain in the fixed tube. The $\left[d_{2}\left(n, n^{\prime}, t\right)\right]^{2}$ does not include the contribution from the non-diffusive part of the CLF. The curves indicate the relationship between $d_{2}^{2}$ and $\mu$ derived in this study. The symbols are for the reptating chain in the fixed tube; see Appendix A.

well as the range of $\varphi>0.5$ for the case of fixed tube) corresponds to a range of time $t<0.5 \tau_{\mathrm{G}} \cdot{ }^{15}$

\section{Evaluation of $\left[\overline{(\Delta \boldsymbol{r})^{2}}\right]$}

As in the fixed tube case, the chain conformations at times 0 and $t$ are characterized by the numbers 
$m_{0}$ and $m$ of the primitive segments out of the left edge of the surviving portion of the dilated tube; see Figures $1 \mathrm{c}$ and $1 \mathrm{~d}$. However, differing from the fixed tube case, the primitive chains at these times are not superimposed with each other even at the portions contained in the surviving part of the dilated tube. This lack of superposition occurs due to the lateral displacement of the segments in the dilated tube in the interval of time from 0 to $t$. For this case, we can evaluate the displacement vector $\Delta \boldsymbol{r}=[\boldsymbol{r}(n, t)-$ $\left.\boldsymbol{r}\left(n^{\prime}, 0\right)\right]$ by dividing the chain motion in two steps, as explained below.

In step 1 , the chain is regarded to move in a fixed (non-dilated) tube of diameter $a$ hypothetically considered around the chain at time 0 . The number of the segments contained in this tube at the time $t$ is identical to that in the dilated tube $\left(=N \varphi^{\prime}\right)$, and the survival fraction of the hypothetical fixed tube at $t$ is given by $\varphi^{\prime}$. The displacement $\Delta \boldsymbol{r}_{(1)}$ in the step 1 is equivalent to those explained in eqs. 4-7. For example, if the $n^{\prime}$-th segment at time 0 and the $n$-th segment at time $t$ are both out of the left edge of the surviving portion of the tube, $\Delta \boldsymbol{r}_{(1)}$ is given by a difference of the vectors $\boldsymbol{r}$ and $\boldsymbol{r}_{0}$ connecting the edge of this portion and the segment positions in these states (cf. Figures $1 \mathrm{~b}$ and $1 \mathrm{~d}$ ). For the other case that the $n$-th and the $n^{\prime}$-th segments are in the surviving portion at times $t$ and $0, \Delta \boldsymbol{r}_{(1)}$ coincides with an end-to-end vector of a Gaussian sequence of $\left|(n-m)-\left(n^{\prime}-m_{0}\right)\right|$ segments (cf. Figures 1a and 1c; the filled circle in Figure 1c indicates the location of the segment after the step 1).

In step 2, the chain is considered to laterally fluctuate in the dilated tube without exhibiting longitudinal motion. If the $n$-th segment is in the surviving portion of the dilated tube after step 1, the $\Delta \boldsymbol{r}_{(2)}$ for step 2 coincides with a displacement $\boldsymbol{D}$ of this segment in an internal cross-section of this portion (shown with the dotted ellipse in Figure 1c). For the other cases, $\Delta \boldsymbol{r}_{(2)}$ is given by a displacement $\boldsymbol{D}$ of a segment located at the edge of the surviving portion; cf. filled circles shown in Figure 1d: In general, this edge segment is not the same segment at the time 0 (before step 1) and the time $t$ (after step 2) because of the longitudinal motion during the step 1 , and $\boldsymbol{D}$ is defined as a vector connecting the positions of the edge segment at these times.

The net displacement $\Delta \boldsymbol{r}$ coincides with a sum of the $\Delta \boldsymbol{r}_{(1)}$ and $\boldsymbol{D}$ for the above steps. ${ }^{15}$ The $\Delta \boldsymbol{r}_{(1)}$ and $\boldsymbol{D}$ are not correlated, and the conditional average of $(\Delta \boldsymbol{r})^{2}$ for the given $m, m_{0}, n$ and $n^{\prime}$ values is obtained as

$$
\left[\overline{(\Delta \boldsymbol{r})^{2}}\right]=\left[\overline{\left(\Delta \boldsymbol{r}_{(1)}\right)^{2}}\right]+\left\langle\boldsymbol{D}^{2}\right\rangle
$$

Here, $\left[\overline{\left(\Delta \boldsymbol{r}_{(1)}\right)^{2}}\right]$ is given by one of eqs. $4-7$ according to the $m, m_{0}, n$ and $n^{\prime}$ values. Since the distribution of the lateral displacement is determined by the dilated tube diameter $a^{\prime}$ and independent of the $m, m_{0}, n$ and $n^{\prime}$ values, the average $\left\langle\boldsymbol{D}^{2}\right\rangle$ can be calculated in the following way. ${ }^{15}$

If the $n$-th segment is in the surviving portion of the dilated tube after step 1 (Figure 1c), the segment position after steps 1 and 2 should be uniformly distributed in the internal cross-section of this tube (shown with the dotted ellipse). Then, $\boldsymbol{D}$ is equivalent to a vector connecting arbitrarily chosen two points in a circular region of the diameter $a^{\prime}-a$, the region available for the center of the $n$-th segment. This equivalence holds also for the other cases where $\boldsymbol{D}$ represents the random displacement of the edge segment. Thus, for all cases, $\left\langle\boldsymbol{D}^{2}\right\rangle$ can be evaluated as ${ }^{15}$

$$
\begin{aligned}
\left\langle\boldsymbol{D}^{2}\right\rangle= & \frac{1}{\pi^{2}\left(\frac{a^{\prime}-a}{2}\right)^{4}} \int \mathrm{d} x \mathrm{~d} x_{0} \mathrm{~d} y \mathrm{~d} y_{0} \\
& \times\left[\left(x-x_{0}\right)^{2}+\left(y-y_{0}\right)^{2}\right] \\
= & \frac{a^{2}}{4}\left[\left(\varphi^{\prime}(t)\right)^{-\alpha / 2}-1\right]^{2}
\end{aligned}
$$

Here, $\left(x_{0}, y_{0}\right)$ and $(x, y)$ are the coordinates of the two points explained above, and the integral is conducted in a range of $\left(x_{0}^{2}+y_{0}^{2}\right)<\left(a^{\prime}-a\right)^{2} / 4$ and $\left(x^{2}+y^{2}\right)<$ $\left(a^{\prime}-a\right)^{2} / 4$. (The DTD relationship $a^{\prime}=a\left[\varphi^{\prime}(t)\right]^{-\alpha / 2}$ has been utilized in eq. 25.) Note that $\left\langle\boldsymbol{D}^{2}\right\rangle$ is independent of $m, m_{0}, n$ and $n_{0}$.

\section{Expression of $d_{2}^{2}$}

The cross displacement function $\left[d_{2}\left(n, n^{\prime}, t\right)\right]^{2}$ for the case of DTD (with $\varphi^{\prime}>0.5 ; \tau_{\mathrm{R}}<t<0.5 \tau_{\mathrm{G}}$ ) can be obtained from eq. 8 with eqs. 24 and 25 . The $\left[\overline{\left.\left(\Delta \boldsymbol{r}_{(1)}\right)^{2}\right]}\right.$ term in eq. 24 , due to the motion of the chain in the hypothetical fixed tube, is equivalent to those in eqs. $4-7$, and the average $\left\langle\overline{\left(\Delta \boldsymbol{r}_{(1)}\right)^{2}}\right\rangle$ is given by eqs. $21-23$ with $\mu(=\varphi)$ therein being replaced by the survival fraction of the dilated tube, $\varphi^{\prime}$.

This $\varphi^{\prime}(t)$ is expressed in terms of the viscoelastic $\mu(t) ; \varphi^{\prime}(t)=[\mu(t)]^{\delta}$ with $\delta=1 /(1+\alpha) \cong 1 / 2$ for the case of DTD. ${ }^{1,2}$ Thus, we finally obtain the following:

$$
\text { For } 0 \leq \theta, \theta^{\prime}<\left(1-\mu^{\delta}\right)
$$

$$
d_{2}^{2} \cong\left\langle R^{2}\right\rangle\left[\gamma^{\prime}+\frac{\left(1-\mu^{\delta}-\theta\right)\left(1-\mu^{\delta}-\theta^{\prime}\right)}{\left(1-\mu^{\delta}\right)^{2}}\left(1-\mu^{\delta}-\frac{\theta+\theta^{\prime}}{2}-\gamma^{\prime}\right)+\frac{\left(\mu^{-\alpha \delta / 2}-1\right)^{2}}{4 N}\right]
$$


For $\mu^{\delta}<\theta, \theta^{\prime} \leq 1$

$$
d_{2}^{2} \cong\left\langle R^{2}\right\rangle\left[\gamma^{\prime}+\frac{\left(\theta-\mu^{\delta}\right)\left(\theta^{\prime}-\mu^{\delta}\right)}{\left(1-\mu^{\delta}\right)^{2}}\left(-\mu^{\delta}+\frac{\theta+\theta^{\prime}}{2}-\gamma^{\prime}\right)+\frac{\left(\mu^{-\alpha \delta / 2}-1\right)^{2}}{4 N}\right]
$$

For all other $\theta, \theta^{\prime}$

$$
d_{2}^{2} \cong\left\langle R^{2}\right\rangle\left[\gamma^{\prime}+\frac{\left(\mu^{-\alpha \delta / 2}-1\right)^{2}}{4 N}\right]
$$

with $\gamma^{\prime}=\left[\left|\theta-\theta^{\prime}\right|^{3}+\left\{\left(1-\mu^{\delta}\right) / 2\right\}^{3}\right]^{1 / 3}, \theta=n / N$, and $\theta^{\prime}=n^{\prime} / N$.

\section{COHERENT INTERMEDIATE SCATTERING FUNCTION}

For a labeled probe chain in a matrix of unlabeled chains of the same molecular weight, the coherent intermediate scattering function $I_{\mathrm{c}}(\boldsymbol{q}, t)$ is related to the motion of the structural units that induce scattering (e.g., nuclei for the neutron scattering). Here, we focus on a chain having labeled monomers only in a particular set of primitive segments. For this partially labeled chain, the $I_{\mathrm{c}}(\boldsymbol{q}, t)$ defined by eq. 3 can be rewritten, under the Gaussian approximation, as

$$
\begin{aligned}
I_{\mathrm{c}}(\boldsymbol{q}, t) & =\frac{1}{g N^{*}} \sum_{j}^{\prime} \sum_{j^{\prime}}^{\prime} \exp \left(-\frac{q^{2}}{6}\left\langle\left[\rho(j, t)-\rho\left(j^{\prime}, 0\right)\right]^{2}\right\rangle\right) \\
& =g N^{*}-\frac{q^{2}}{6 g N^{*}} \sum_{j}^{\prime} \sum_{j^{\prime}}^{\prime}\left\langle\left[\rho(j, t)-\rho\left(j^{\prime}, 0\right)\right]^{2}\right\rangle+O\left(q^{4}\right) .
\end{aligned}
$$

In eq. $29, N^{*}$ is the number of labeled primitive segments, and the summation $\sum^{\prime}$ is taken for the labeled monomers.

The displacement of these monomers are represented in terms of the displacement of the primitive segment. For the monomers $j$ and $j^{\prime}$ belonging to the $n$ th and $n^{\prime}$-th primitive segment, respectively, we find their cross-displacement $\left\langle\left[\rho(j, t)-\rho\left(j^{\prime}, 0\right)\right]^{2}\right\rangle$ to be given by $\left\langle\left[\Delta \rho_{C M}\left(n, n^{\prime}, t\right)+\Delta \rho_{f}(j)+\Delta \rho_{f}\left(j^{\prime}\right)\right]^{2}\right\rangle$. Here, $\Delta \rho_{C M}\left(n, n^{\prime}, t\right)$ is the displacement of the center of mass of $n$-th primitive segment at $t$ with respect to the center of mass of $n^{\prime}$-th primitive segment at time 0. $\Delta \rho_{f}(j)$ and $\Delta \rho_{f}\left(j^{\prime}\right)$ represent fluctuation of $j$-th and $j^{\prime}$-th monomer within $n$-th and $n^{\prime}$-th primitive segments, respectively. The center of mass displacement $\Delta \rho_{C M}$ is uncorrelated with this fluctuation. Hence, $\left\langle\left[\rho(j, t)-\rho\left(j^{\prime}, 0\right)\right]^{2}\right\rangle=\left\langle\left[\Delta \rho_{C M}\left(n, n^{\prime}, t\right)\right]^{2}\right\rangle+$ $2\left\langle\left[\Delta \boldsymbol{\rho}_{f}\right]^{2}\right\rangle$. Since the monomeric segment is much smaller than the primitive segment, we may approximate $\left\langle\left[\Delta \boldsymbol{\rho}_{f}\right]^{2}\right\rangle$ as a mean-square separation of two points randomly chosen in a sphere of diameter $a$. This leads to $\left\langle\left[\Delta \rho_{f}\right]^{2}\right\rangle=3 a^{2} / 10 .^{15}$

The $\left[d_{2}\left(n, n^{\prime}, t\right)\right]^{2}$ calculated in eqs. $21-23$ and/or in eqs. $26-28$ is the mean square displacement not contributed from the nondiffusive part of the contour length fluctuation (CLF). This $d_{2}^{2}$ is equal to $\left\langle\left[\Delta \boldsymbol{\rho}_{C M}\left(n, n^{\prime}, t\right)\right]^{2}\right\rangle-d_{2, \mathrm{CLF}}^{2}$. Here, the mean-square displacement due only to the nondiffusive part of CLF, $d_{2, \mathrm{CLF}}^{2}$, is defined as a quantity that does not include a term corresponding to the initial condition, $\left\langle\left[\boldsymbol{r}(n, 0)-\boldsymbol{r}\left(n^{\prime}, 0\right)\right]^{2}\right\rangle=a\left|n-n^{\prime}\right|$. (This term is included in $\left[d_{2}\left(n, n^{\prime}, t\right)\right]^{2}$; see eqs. $21-23$ and 26-28 with $\mu=1$ (at $t=0)$.)

For the Rouse-type CLF process, this $d_{2, \mathrm{CLF}}^{2}$ can be expressed as ${ }^{7}$

$$
\left[d_{2, \mathrm{CLF}}\left(n, n^{\prime}, t\right)\right]^{2} \cong a^{2}\left\{\sum_{p=1}^{\infty} \frac{2 N}{3 \pi^{2} p^{2}}\left[\cos ^{2}\left(\frac{p \pi n}{N}\right)+\cos ^{2}\left(\frac{p \pi n^{\prime}}{N}\right)-2 \cos \left(\frac{p \pi n}{N}\right) \cos \left(\frac{p \pi n^{\prime}}{N}\right) \exp \left(-\frac{t p^{2}}{\tau_{\mathrm{R}}}\right)\right]\right\}^{1 / 2}
$$

At $t>\tau_{\mathrm{R}}$ where the CLF process is completed, the exponential terms can be safely neglected. The summation over two squared cosine terms can be carried out analytically; see Appendix B for a detailed calculation including the derivation of eq. 30 . The calculation gives t-independent $d_{2, \mathrm{CLF}}^{2}$

$$
\left[d_{2, \mathrm{CLF}}\left(n, n^{\prime}\right)\right]^{2} \cong \frac{\left\langle R^{2}\right\rangle}{(6 N)^{1 / 2}}\left[\frac{1}{3}+2\left(\theta-\frac{1}{2}\right)^{2}+2\left(\theta^{\prime}-\frac{1}{2}\right)^{2}\right]^{1 / 2} \quad \text { at } t>\tau_{\mathrm{R}}
$$


with $\theta=n / N$ and $\theta^{\prime}=n^{\prime} / N$. This method of calculating $d_{2, \mathrm{CLF}}^{2}$ is more accurate than the previous method ${ }^{15}$ utilizing the pre-average of the squared cosine terms in eq. 30 .

$I_{\mathrm{c}}(\boldsymbol{q}, t)$ can thus be written as

$$
I_{\mathrm{c}}(\boldsymbol{q}, t)=I_{\mathrm{c}}(\mathbf{0}, t)\left[1-\frac{q^{2}}{6}\left\{\frac{3 a^{2}}{5}+\frac{1}{N^{* 2}} \sum_{n}^{\prime} \sum_{n^{\prime}}^{\prime}\left(\left[d_{2}\left(n, n^{\prime}, t\right)\right]^{2}+\left[d_{2, \mathrm{CLF}}\left(n, n^{\prime}\right)\right]^{2}\right)\right\}\right]+O\left(q^{4}\right) \quad \text { at } 0.5 \tau_{\mathrm{G}}>t>\tau_{\mathrm{R}}
$$

with $I_{\mathrm{c}}(\mathbf{0}, t)=g N^{*}$ and the summation $\sum^{\prime}$ being taken for the labeled primitive segments. Here $\left[d_{2}\left(n, n^{\prime}, t\right)\right]^{2}$ is to be substituted from eqs. 21-23 for the case of fixed tube and from eqs. 26-28 for the case of DTD, and $d_{2, \mathrm{CLF}}^{2}$ is to be taken from eq. 31. Equations 21-23, 26-28 and 31 do not depend on $t$ explicitly so that this substitution allows us to erase the explicit $t$ dependence and introduce the $\mu$ dependence in $I_{\mathrm{c}}$. Thus eq. 32 gives the relationship between $I_{\mathrm{c}}$ and $\mu$ for the cases of the fixed and dilated tube.

\section{EXPERIMENTAL TEST OF DTD PICTURE AT SHORT TIMES}

Here, we consider a chain having the scattering label (such as the isotope label) only in two primitive segments ( $n_{1}$-th and $n_{2}$-th segments; $N^{*}=2$ ) each containing $g$ monomeric segments. For this partially labeled probe placed in a matrix of unlabeled chains of the same molecular weight, dynamic scattering experiments allow us to measure the coherent intermediate scattering function $I_{\mathrm{c}}(\boldsymbol{q}, t)$. From eq. 32, this $I_{\mathrm{c}}(\boldsymbol{q}, t)$ is related to the normalized viscoelastic relaxation function $\mu(t)$ as

$$
\frac{I_{\mathrm{c}}(\mathbf{0}, t)-I_{\mathrm{c}}(\boldsymbol{q}, t)}{g q^{2}\left\langle R^{2}\right\rangle}=\frac{1}{5 N}+J\left(n_{1}, n_{2}, t\right)+J_{\mathrm{CLF}}\left(n_{1}, n_{2}\right) \quad \text { at low } q \text { and at } \tau_{\mathrm{R}}<t<0.5 \tau_{\mathrm{G}}
$$

with

$$
J\left(n_{1}, n_{2}, t\right)=\frac{1}{12\left\langle R^{2}\right\rangle}\left\{2\left[d_{2}\left(n_{1}, n_{2}, t\right)\right]^{2}+\left[d_{2}\left(n_{1}, n_{1}, t\right)\right]^{2}+\left[d_{2}\left(n_{2}, n_{2}, t\right)\right]^{2}\right\}
$$

and

$$
J_{\mathrm{CLF}}\left(n_{1}, n_{2}\right)=\frac{1}{12\left\langle R^{2}\right\rangle}\left\{2\left[d_{2, \mathrm{CLF}}\left(n_{1}, n_{2}\right)\right]^{2}+\left[d_{2, \mathrm{CLF}}\left(n_{1}, n_{1}\right)\right]^{2}+\left[d_{2, \mathrm{CLF}}\left(n_{2}, n_{2}\right)\right]^{2}\right\}
$$

The $d_{2}^{2}$ terms, determining the $\mu$ dependence of $I_{\mathrm{c}}$, are to be substituted from eqs. $21-23$ or eqs. $26-28$, and the $d_{2, \mathrm{CLF}}^{2}$ terms from eq. 31 .

In Figure 3, we show some example plots of the relationship between the experimentally measurable quantities $\left[I_{\mathrm{c}}(\mathbf{0}, t)-I_{\mathrm{c}}(\boldsymbol{q}, t)\right] / g q^{2}\left\langle R^{2}\right\rangle$ and $\mu$ for three sets of the label location $\left(n, n^{\prime}\right)$. The dotted curves indicate the contribution of the $J$ term (eq. 34) to the reduced scattering function $\left[I_{\mathrm{c}}(\mathbf{0}, t)-I_{\mathrm{c}}(\boldsymbol{q}, t)\right] / g q^{2}\left\langle R^{2}\right\rangle$. For the moderately separated $n$ and $n^{\prime}$ values chosen here, the curves are close to the plots (i.e., the $J$ term dominates this function). Thus, the comparison of the $I_{\mathrm{c}}$ data and eq. 33 can be made without being disturbed by a (small) uncertainty in the calculation of $d_{2, \mathrm{CLF}}^{2}$ (due to the use of the Rouse expression without the exponential decay terms; eqs. 30 and 31 ).

As seen in Figure 3, the $\mu$ dependence of $\left[I_{\mathrm{c}}(\mathbf{0}, t)-\right.$ $\left.I_{\mathrm{c}}(\boldsymbol{q}, t)\right] / g q^{2}\left\langle R^{2}\right\rangle$ is significantly different for the two cases of the fixed tube and DTD. This difference allows us to test the DTD picture at short $t$ (but still longer than $\left.\tau_{\mathrm{R}}\right)$ through comparison of the $\left[I_{\mathrm{c}}(\mathbf{0}, t)-\right.$ $\left.I_{\mathrm{c}}(\boldsymbol{q}, t)\right] / g q^{2}\left\langle R^{2}\right\rangle$ and $\mu$ data for the partially labeled dilute probe in monodisperse systems.

\section{CONCLUDING REMARKS}

Considering just the Gaussian feature of the chain, we have analyzed the relationship between meansquare displacement of the $n$-th segment at time $t$ with respect to the $n^{\prime}$-th segment at time $0,\left[d_{2}\left(n, n^{\prime}, t\right)\right]^{2}$ (not contributed from the non-diffusive part of CLF). This analysis gave a relationship between $d_{2}^{2}$ and the normalized viscoelastic relaxation function $\mu(t)$ at short $t$ (but still longer than the Rouse time $\tau_{R}$ for CLF) for the cases of the fixed tube and DTD. This relationship was significantly different for these cases, enabling us to utilize the data of the coherent intermediate scattering function $I_{\mathrm{c}}(\boldsymbol{q}, t)$ and $\mu(t)$ to test the DTD picture at short $t$.

Acknowledgment. AKRP acknowledges, with thanks, financial support from JSPS under its PostDoctoral Research Program (P01279). 

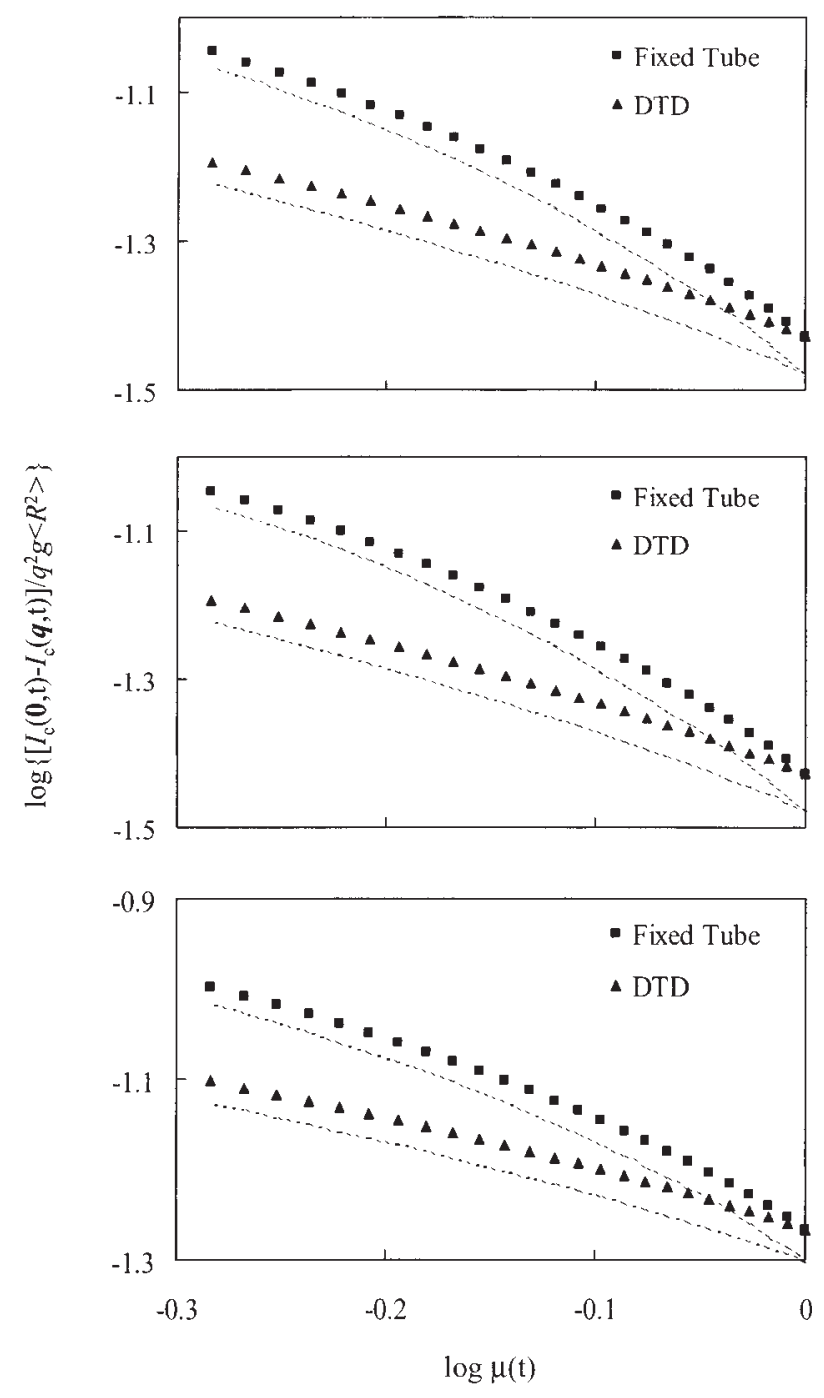

Figure 3. Plots of the reduced scattering function $\left[I_{\mathrm{c}}(\mathbf{0}, t)-\right.$ $\left.I_{\mathrm{c}}(\boldsymbol{q}, t)\right] / g q^{2}\left\langle R^{2}\right\rangle$ against the viscoelastic relaxation function $\mu$ for the cases of fixed tube and DTD (eq. 33). Dotted curves show the contribution of the $J$ term in eq. 33 (representing the chain motion without CLF). The two labeled segments are at (a) $n=$ $0.3 N, n^{\prime}=0.5 N$ (b) $n=0.4 N, n^{\prime}=0.6 N$ and (c) $n=0.4 N, n^{\prime}=$ $0.7 N . N=500$ for all cases.

\section{APPENDIX}

\section{A. Cross Displacement from Reptation Theory}

For the primitive chain having a constant length (without CLF) in the fixed tube, reptation is the only possible mode of motion. For this chain, the cross displacement function $\left[d_{2}\left(n, n^{\prime}, t\right)\right]^{2}=\langle[\boldsymbol{r}(n, t)-$ $\left.\left.\boldsymbol{r}\left(n^{\prime}, 0\right)\right]^{2}\right\rangle$ (denoted as $\phi\left(s, s^{\prime} ; t\right)$ in the textbook by Doi and Edwards ${ }^{7}$ ) obeys the diffusion equation,

$$
\frac{\partial}{\partial t} d_{2}^{2}=D_{c} \frac{\partial^{2}}{\partial n^{2}} d_{2}^{2}
$$

where $D_{c}$ is the curvilinear diffusion coefficient. Under appropriate initial and boundary conditions (representing the Gaussian conformation and the tension balance at the chain ends), eq. A.1 gives

$$
\begin{aligned}
& {\left[d_{2}\left(n, n^{\prime}, t\right)\right]^{2}=\left|n-n^{\prime}\right| a^{2}+2 \frac{D_{c} t}{N}} \\
& \quad+\sum_{p=1}^{\infty} \frac{4 N a^{2}}{p^{2} \pi^{2}} \times \cos \left(\frac{p \pi n}{N}\right) \\
& \quad \times \cos \left(\frac{p \pi n^{\prime}}{N}\right)\left\{1-\exp \left(-\frac{p^{2} t}{\tau_{\text {rep }}}\right)\right\}
\end{aligned}
$$

with $\tau_{\text {rep }}=N^{2} / \pi^{2} D_{c}$.

For the shear orientation function $S(n, t)=$ $a^{-2}\langle\boldsymbol{u}(n, t) \boldsymbol{u}(n, t)\rangle_{x y}$ with $\boldsymbol{u}=\partial \boldsymbol{r}(n, t) / \partial n$, the same type of diffusion equation holds. ${ }^{7}$ Under the initial and boundary conditions representing uniform orientation at $t=0$ and rapid randomization at the chain ends, $S(n, t)$ is solved to give

$$
\mu(t)=\frac{G(t)}{G_{N}}=\sum_{p=\text { odd }} \frac{8}{p^{2} \pi^{2}} \exp \left(-\frac{t p^{2}}{\tau_{\text {rep }}}\right)
$$

( $\mu(t)$ is obtained as an integral of $S(n, t)$ with respect to $n)$. In Figure $2, d_{2}^{2}$ given by eq. A.2 is plotted against this $\mu$. (In the range of $\mu(t)>0.5(t<$ $\left.0.5 \tau_{\text {rep }}\right)$, the Fickian diffusion represented by the $2 D_{c} t / N$ term in eq. A. 2 does not significantly contribute to $d_{2}^{2}$.)

\section{B. Dynamics of the Contour Length Fluctuation}

For examining the dynamics of the contour length fluctuation, we follow the Doi-Edwards treatment ${ }^{7}$ to use the Rouse model. Let $s(n, t)$ be the curvilinear coordinate of the $n$-th Rouse segment (of size $a$ ) at time $t$ measured from a fixed point on the tube. This $s(n, t)$ is expanded into its eigenmodes,

$$
s(n, t)=Y_{0}(t)+2 \sum_{p=1}^{\infty} Y_{p}(t) \cos \left(\frac{p \pi n}{N}\right)+\frac{n}{N} L_{\mathrm{eq}}
$$

Here $L_{\mathrm{eq}}(=N a)$ is the average length of the chain. The normal coordinates $Y_{p}(p \geq 0)$ are related to $s(n, t)$ as

$$
Y_{p}(t)=\frac{1}{N} \int_{0}^{N} \mathrm{~d} n\left(s(n, t)-\frac{n L_{\mathrm{eq}}}{N}\right) \cos \left(\frac{p \pi n}{N}\right)
$$

The time correlation functions for $Y_{p}$ are given by

$$
\begin{gathered}
\left\langle\left[Y_{0}(t)-Y_{0}(0)\right]^{2}\right\rangle=2 \frac{k_{\mathrm{B}} T}{N \zeta} t \\
\left\langle Y_{p}(t) Y_{q}(0)\right\rangle=\frac{N a^{2} \delta_{p q}}{6 \pi^{2} p^{2}} \exp \left(\frac{-t p^{2}}{\tau_{\mathrm{R}}}\right)
\end{gathered}
$$

Here, $\zeta$ is the friction coefficient of the Rouse segment, and $\tau_{\mathrm{R}}$ is the Rouse time.

The curvilinear mean-square displacement of the $n$ - 
th segment at time $t$ with respect to $n^{\prime}$-th segment at time 0 is given by

$$
\begin{aligned}
\left\langle\left[s(n, t)-s\left(n^{\prime}, 0\right)\right]^{2}\right\rangle= & \left\langle\left[Y_{0}(t)-Y_{0}(0)\right]^{2}\right\rangle+4\left\langle\sum_{p=1}^{\infty}\left[Y_{p}(t) \cos \left(\frac{p \pi n}{N}\right)-Y_{p}(0) \cos \left(\frac{p \pi n^{\prime}}{N}\right)\right]^{2}\right\rangle+a^{2}\left(n-n^{\prime}\right)^{2} \\
= & \frac{a^{2}}{6} \sum_{p=1}^{\infty} \frac{4 N}{p^{2} \pi^{2}}\left[\cos ^{2}\left(\frac{p \pi n}{N}\right)+\cos ^{2}\left(\frac{p \pi n^{\prime}}{N}\right)-2 \cos \left(\frac{p \pi n}{N}\right) \cos \left(\frac{p \pi n^{\prime}}{N}\right) \exp \left(-\frac{t p^{2}}{\tau_{\mathrm{R}}}\right)\right] \\
& +a^{2}\left(n-n^{\prime}\right)^{2}+\frac{2 k_{\mathrm{B}} T t}{N \zeta}
\end{aligned}
$$

Since the $2 k_{\mathrm{B}} T t / N \zeta$ term represents the Fickian diffusion along the tube, the $d_{2, \mathrm{CLF}}^{2}$ analyzed in this study is related to eq. B.5 without this term. Further, the $a^{2}\left(n-n^{\prime}\right)^{2}$ term giving the initial condition, $d_{2, \mathrm{CLF}}^{2}=a\langle[s(n, t)-$ $\left.\left.s\left(n^{\prime}, 0\right)\right]^{2}\right\rangle^{1 / 2}=a^{2}\left|n-n^{\prime}\right|$ at $t=0$, is not to be included in our calculation because this condition is already accounted in $d_{2}^{2}$ (see eqs. 21-23 and 26-28). Thus, for the time $t>\tau_{\mathrm{R}}$ of our interest where the exponential terms are small and negligible, the relevant curvilinear mean-square displacement can be written as

$$
\left.\left\langle\left[s(n, t)-s\left(n^{\prime}, 0\right)\right]^{2}\right\rangle\right|_{\text {non-diffusive CLF }}=\frac{a^{2}}{6} \sum_{p=1}^{\infty} \frac{4 N}{p^{2} \pi^{2}}\left[1+\frac{1}{2} \cos \left(\frac{2 p \pi n}{N}\right)+\frac{1}{2} \cos \left(\frac{2 p \pi n^{\prime}}{N}\right)\right]
$$

Utilizing a mathematical formula ${ }^{17} \sum_{p=1}^{\infty} \frac{1}{p^{2}} \cos (p x)=\frac{1}{4}(x-\pi)^{2}-\frac{\pi^{2}}{12} \quad$ (for $0 \leq x \leq 2 \pi$ ), we can rewrite eq. B.6 as

$$
\left.\left\langle\left[s(n, t)-s\left(n^{\prime}, 0\right)\right]^{2}\right\rangle\right|_{\text {non-diffusive CLF }}=\frac{a^{2} N}{6}\left[\frac{1}{3}+2\left(\frac{n}{N}-\frac{1}{2}\right)^{2}+2\left(\frac{n^{\prime}}{N}-\frac{1}{2}\right)^{2}\right]
$$

From eq. B.7 the contribution of the non-diffusive part of CLF to the cross-displacement function is obtained as

$$
\left[d_{2, \mathrm{CLF}}\left(n, n^{\prime}\right)\right]^{2}=a\left\langle\left[s(n, t)-s\left(n^{\prime}, 0\right)\right]^{2}\right\rangle^{1 / 2}=a^{2}\left(\frac{N}{6}\right)^{1 / 2}\left[\frac{1}{3}+2\left(\theta-\frac{1}{2}\right)^{2}+2\left(\theta^{\prime}-\frac{1}{2}\right)^{2}\right]^{1 / 2}
$$

with $\theta=n / N$ and $\theta^{\prime}=n^{\prime} / N$.

\section{REFERENCES}

1. G. Marrucci, J. Polym. Sci., Polym. Phys. Ed., 23, 159 (1985).

2. H. Watanabe, Prog. Polym. Sci., 24, 1253 (1999).

3. R. C. Ball and T. C. B. McLeish, Macromolecules, 22, 1911 (1989).

4. S. T. Milner and T. C. B. McLeish, Macromolecules, 30, 2159 (1997).

5. S. T. Milner and T. C. B. McLeish, Macromolecules, 31, 7479 (1998).

6. S. T. Milner and T. C. B. McLeish, Phys. Rev. Lett., 81, 725 (1998).

7. M. Doi and S. F. Edwards, "The Theory of Polymer Dynamics", Clarendon, Oxford, 1986.

8. Y. Matsumiya, H. Watanabe, and K. Osaki, Macromolecules, 33, 499 (2000).
9. H. Watanabe, Y. Matsumiya, and K. Osaki, J. Polym. Sci., Part B: Polym. Phys., 38, 1024 (2000).

10. H. Watanabe, Kor.-Aust. Rheol. J., 13, 205 (2001).

11. H. Watanabe, Y. Matsumiya, and T. Inoue, Macromolecules, 35, 2339 (2002).

12. Y. Matsumiya and H. Watanabe, Macromolecules, 34, 5702 (2001).

13. T. Inoue, H. Okamoto, and K. Osaki, Macromolecules, 24, 5670 (1991).

14. T. Inoue, H. Onogi, and K. Osaki, J. Polym. Sci., Part B: Polym. Phys., 37, 389 (1999).

15. H. Watanabe and A. K. R. Paul, Macromolecules, 36, 2526 (2003).

16. T. Kanaya and K. Kaji, Adv. Polym. Sci., 87, 154 (2001).

17. S. Moriguchi, K. Udagawa, and S. Hitotsumatsu, "Sugaku Koshiki II (Mathematical Formula II)", Iwanami, Tokyo, 1986. 